\title{
Estimation of Surface Excess from Compositional Depth Profiles of Iron-base Alloys Passivated in Sulfuric Acid Solution*
}

\author{
By Masahiro SEO** and Norio SATO**
}

\begin{abstract}
Synopsis
A differential method for obtaining the exact composition profiles in depth by use of AES and argon ion sputter-etching was applied to the analyses of $\mathrm{Fe}-30 \mathrm{Cr}$ alloy, 304- and 316-stainless steels potentiostatically passivated in deaerated $0.5 \mathrm{M}$ sulfuric acid solution. From the measured composition profiles in depth, the surface excess of components, $\Gamma_{\mathrm{Cr}}, \Gamma_{\mathrm{Ni}}$, $\Gamma_{\mathrm{O}}$ and $\Gamma_{\mathrm{S}}$ on the dividing surface with $\Gamma_{\mathrm{Fe}} \approx 0$ was estimated as a function of anodic potential. The surface excess changes depending on the species of alloys as follows: $\Gamma_{\mathrm{Cr}}=1 \sim 3 \times 10^{15}$ atoms $/ \mathrm{cm}^{2}, \Gamma_{\mathrm{Ni}}=4 \sim$ $8 \times 10^{14}$ atoms $/ \mathrm{cm}^{2}, \Gamma_{\mathrm{O}}=3 \sim 6 \times 10^{15} \mathrm{atoms} / \mathrm{cm}^{2}$ and $\Gamma_{\mathrm{S}}=2 \sim 8 \times 10^{14}$ atoms $/ \mathrm{cm}^{2}$. Specially, the values of $\Gamma_{\mathrm{C}_{\mathrm{r}}}$ and $\Gamma_{\mathrm{O}}$ on 316-stainless steel are smaller than those on 304-stainless steel, indicating that molybdenum promotes no enrichment of chromium in the passive film. The corrosion resistivity of the alloys is discussed by taking into account of both microbreakdown of the film and repassivation of the substrate alloy at breakdown sites. It is concluded that the beneficial effect of molybdenum on the corrosion resistivity can be attributed not to the stability of the film itself but to the acceleration of repassivation process at breakdown sites.
\end{abstract}

\section{Introduction}

Surface enrichment of certain alloying components is expected to play a vital role on the corrosion resistance of iron-base alloys. The chromium enrichment $^{1)}$ in the passive films on corrosion-resistive ironchromium alloys has been revealed from the composition profiles in depth of these films measured by AES and argon ion sputter-etching techniques. The conventional composition profiles in depth as measured by AES, however, necessarily involve in part the contribution from the subsequent layers because the Auger electrons have an escape depth $(0.5 \sim 3.0 \mathrm{~nm})$ and hence can not exhibit the exact composition of successive layers. Recently, F. Pons et al. ${ }^{2,3)}$ have developed a differential method to obtain the exact composition of successive layers by eliminating the influence of the subsequent layers.

In the present study, this differential method was applied to the surface analyses of $\mathrm{Fe}-30 \mathrm{Cr}$ alloy, 304and 316- stainless steels passivated potentiostatically in deaerated $0.5 \mathrm{M}$ sulfuric acid solution. The surface excess $\Gamma_{1}$, of alloying and environmental components of chromium, nickel, oxygen and sulfur on a reference plane (the so-called dividing surface) with
$\Gamma_{\mathrm{Fe}} \approx \mathrm{O}$ was estimated as a function of anodic potential from the exact composition profiles in depth. Furthermore, the corrosion resistance of these alloys were discussed in terms of the surface excess of alloying elements.

\section{Experimental}

\section{Specimen Preparation}

The specimens were of $\mathrm{Fe}-30 \mathrm{Cr}$ alloy plates $(1.0 \mathrm{~cm} \times 1.0 \mathrm{~cm} \times 0.15 \mathrm{~cm})$ and of $304-$ and $316-$ stainless steel plates $(1.0 \mathrm{~cm} \times 1.0 \mathrm{~cm} \times 0.2 \mathrm{~cm})$, whose exact compositions are listed in Table 1. The Fe$30 \mathrm{Cr}$ alloy and the stainless steels were respectively vacuum-annealed at $850^{\circ} \mathrm{C}$ for $15 \mathrm{~min}$ and at $1100^{\circ} \mathrm{C}$ for $30 \mathrm{~min}$ and then quenched in water. The specimens were polished with emery papers, subsequently with $\alpha-\mathrm{Al}_{2} \mathrm{O}_{3}$ abrasives $(0.5 \mu \mathrm{m})$, and washed with acetone using ultrasonic techniques. The electrolyte solution used was $0.5 \mathrm{M}$ sulfuric acid solution completely deaerated in solution reservoirs by purified nitrogen gas before the introduction into an electrolytic cell.

The polished specimen was cathodically reduced at a constant current density of $10 \mu \mathrm{A} / \mathrm{cm}^{2}$ for $5 \mathrm{~min}$ in $0.5 \mathrm{M}$ sulfuric acid solution. The solution was then renewed and the specimen was passivated potentiostatically at a given anodic potential for one hour. After one-hour passivation, the specimen was washed with double-distilled water, dried with ethanol under nitrogen atomosphere and stored in a glass tube filled with liquid nitrogen to prevent further oxidation in air and compositional change during storage.

\section{AES Measurements}

The Auger chamber (PHI, 540A), in which the specimen was set on a sample holder, was evacuated to a vacuum better than $2.7 \times 10^{-7} \mathrm{~Pa}$. The specimen was cooled down to a temperature (about $-150^{\circ} \mathrm{C}$ ) by flowing liquid nitrogen continuously into the sample holder. The sputter-etching of the specimen was performed with a $500 \mathrm{eV}$ ion beam having

Table 1. The composition of the iron-base alloys (wt \%)

\begin{tabular}{lllllrllllll} 
& \multicolumn{1}{c}{$\mathrm{C}$} & \multicolumn{1}{c}{$\mathrm{Si}$} & $\mathrm{Mn}$ & $\mathrm{P}$ & $\mathrm{S}$ & $\mathrm{Ni}$ & $\mathrm{Cr}$ & $\mathrm{Mo}$ & $\mathrm{Cu}$ & $\mathrm{Co}$ & $\mathrm{Fe}$ \\
\hline Fe-30Cr & 0.0043 & 0.0092 & 0.002 & 0.002 & $<0.001$ & 0.02 & 29.0 & 0.008 & $<0.01$ & 0.020 & Balance \\
SUS 304 & 0.051 & 0.76 & 0.97 & 0.027 & 0.006 & 9.23 & 18.36 & 0.07 & 0.08 & - & Balance \\
SUS 316 & 0.049 & 0.68 & 1.37 & 0.032 & 0.007 & 11.20 & 17.21 & 2.20 & 0.33 & - & Balance
\end{tabular}

* Originally published in Corrosion Engineering (Boshoku Gijutsu), 27 (1978), 647, in Japanese. English version received February 23, 1979.

** Faculty of Engineering, Hokkaido University, Kita-ku, Sapporo 060. 
an ion current of about $5 \mu \mathrm{A} / \mathrm{cm}^{2}$ at an argon pressure of $6.7 \times 10^{-3} \mathrm{~Pa}$. Under this condition, chromium and iron were sputtered at the same yield from a series of $\mathrm{Fe}-\mathrm{Cr}$ alloy substrates. The sputtering rate $^{4)}$ of the passive films on Fe-30Cr alloy was estimated to be $0.15 \mathrm{~nm} / \mathrm{min}$ from the comparison between the sputtering time required to remove a film completely and the ellipsometrically estimated film thickness. The time-variation in Auger spectra of the specimen during sputter-etching was measured by using a primary $2 \mathrm{kV}, 40 \mu \mathrm{A}$ electron beam with a diameter of about $50 \mu \mathrm{m}$ and a $4 \mathrm{~V}$ modulation amplitude at a frequency of $30 \mathrm{kHz}$.

\section{Differential Method}

According to F. Pons et al., ${ }^{2,3)}$ the number of atoms of component $\mathrm{I}$ in the uppermost surface layer (the first atomic layer) is given by,

$$
n_{I}^{(1)}=\left(H_{I}^{(0)}-k_{I} H_{I}^{(1)}\right) / \alpha_{I}
$$

where $H_{I}^{(0)}$ is the initial Auger peak to peak height of component I before sputter-etching, $H_{I}^{(1)}$ the Auger peak to peak height of the component I obtained after sputter-etching of the first atomic layer, $k_{I}$ the attenuation coefficient of component I and $\alpha_{I}$ the sensitivity coefficient of component I. In general, the number of atoms of component $\mathrm{I}$ in the $i$-th atomic layer is also given by,

$$
n_{I}^{(i)}=\left(H_{I}^{(i-1)}-k_{I} H_{I}^{(i)}\right) / \alpha_{I}
$$

In Eqs. (1) and (2), it is assumed that the Auger peak to peak height is proportional to the corresponding Auger current (i.e. surface concentration) and that the layer by layer-sputter etching is performed uniformly at a constant rate. The attenuation coefficient of component I, $k_{I}$, can be estimated from the following relationship:

$$
k_{I}=\exp \left\{-d\left(\frac{1}{\lambda_{I} \cos \theta_{1}}+\frac{1}{\lambda_{p} \cos \theta_{2}}\right)\right\}
$$

where $d$ is the thickness of each atomic layer, $\lambda_{I}$ the escape depth of Auger electron of component I, $\lambda_{p}$ the mean free path of the primary electron in the specimen, $\theta_{1}$ the acceptance angle of Auger electron in the analyser, and $\theta_{2}$ the emission angle of the primary electron to the specimen.
For determination of $k_{I}$, the values of $d, \lambda_{I}, \lambda_{p}, \cos$ $\theta_{1}$ and $\cos \theta_{2}$ must be known. $d=0.3 \mathrm{~nm}$ was chosen as the thickness of each atomic layer in the anodic oxide film because the monolayer-thickness of adsorbed oxygen had been reported to be approximately $0.3 \mathrm{~nm} .^{5)}$ The escape depth of Auger electron of component I, $\lambda_{I}$, was estimated from the proportional relationship between $\lambda_{I}$ and the square root of the Auger electron energy by assuming the escape depth of the Auger electron of oxygen $(510 \mathrm{eV})$ to be $\lambda_{0}=$ $0.80 \mathrm{~nm}$. According to Kanter's results, ${ }^{6)} \lambda_{p}$ depends on the energy of the primary electron. $\lambda_{p}=3.0 \mathrm{~nm}$ was assumed for the energy of the primary electron $(2 \mathrm{keV})$ used in this experiment. The geometrical configuration between the specimen holder, the analyser and the electron gun gives the values of $\cos$ $\theta_{1} \approx 0.75$ and $\theta_{2}=60^{\circ}$. The values of $k_{I}$ can thus be calculated for $\mathrm{Fe}(651 \mathrm{eV}), \operatorname{Cr}(529 \mathrm{eV}), \mathrm{Ni}(848 \mathrm{eV})$, $\mathrm{O}(510 \mathrm{eV})$ and $\mathrm{S}(152 \mathrm{eV})$, as listed in the second column of Table 2 .

For the determination of $\alpha_{I}$, iron was taken as a reference material. The relative sensitivity coefficients of chromium and nickel, $\alpha_{\mathrm{Cr}}$ and $\alpha_{\mathrm{Ni}}$, were obtained by comparing the Auger peak to peak heights of pure metallic iron, $H_{\mathrm{Fe}}^{\circ}(651 \mathrm{eV})$, chromium $H_{\mathrm{Cr}}^{\circ}$ $(529 \mathrm{eV})$ and nickel $H_{\mathrm{Ni}}^{\circ}(848 \mathrm{eV})$ measured directly under the same analytical condition. The relative sensitivity coefficient of oxygen, $\alpha_{0}$, was obtained from our previous Auger results ${ }^{7}$ of thick iron oxide films with a variety of stoichiometric ratio, $\mathrm{O} / \mathrm{Fe}$. Furthermore, the relative sensitivity coefficient, $\alpha_{\mathrm{S}}$, was estimated from the values of sensitivity coefficients described elsewhere. ${ }^{8)}$ The values of $\alpha_{I}$ thus estimated are listed in the third column of Table 2.

\section{Results and Discussion}

Figure 1 shows the Auger spectra of 316-stainless steel surface passivated at $0.545 \mathrm{~V}$ (NHE) for one hour in $0.5 \mathrm{M}$ sulfuric acid solution. The Auger

Table 2. $k_{\mathrm{I}}$ and $\alpha_{\mathrm{I}}$ for component I

\begin{tabular}{cccccc}
$\mathrm{I}$ & $\mathrm{Fe}(651)$ & $\mathrm{Cr}(529)$ & $\mathrm{Ni}(848)$ & $\mathrm{O}(510)$ & $\mathrm{S}(152)$ \\
\hline$k_{I}$ & 0.53 & 0.50 & 0.56 & 0.50 & 0.33 \\
$\alpha_{I}$ & 1.00 & 1.60 & 0.92 & 2.20 & 6.40
\end{tabular}

Fig. 1.

Auger spectra of the 316-stainless steel anodically oxidized for one hour at $0.545 \mathrm{~V}$ (NHE) in $0.5 \mathrm{M}$ sulfuric acid solution

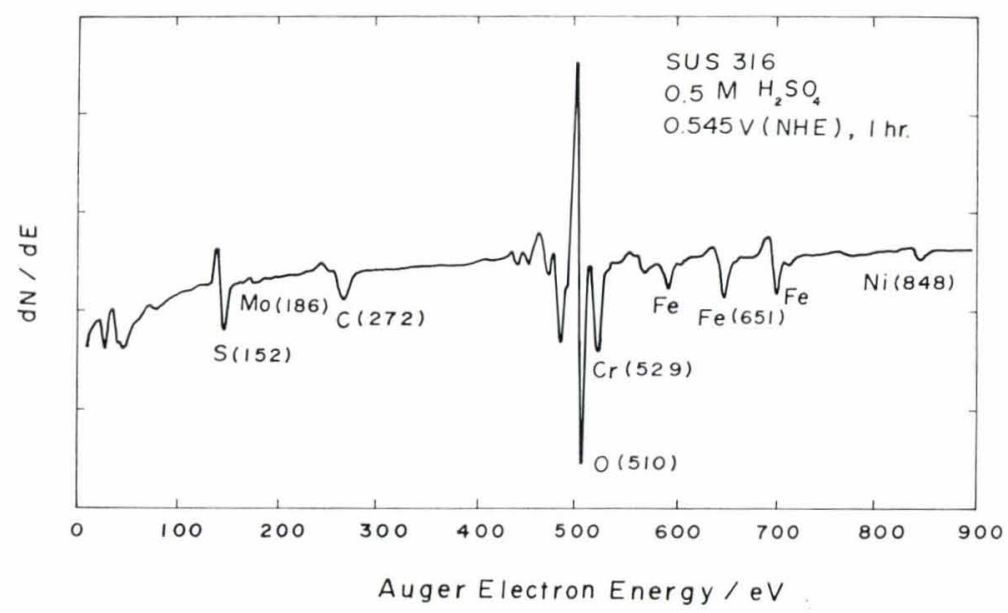

Research Article 
peak to peak heights of $\mathrm{Fe}(651 \mathrm{eV}), \operatorname{Cr}(529 \mathrm{eV})$, $\mathrm{Ni}(848 \mathrm{eV}), \mathrm{Mo}(186 \mathrm{eV}), \mathrm{O}(510 \mathrm{eV})$ and $\mathrm{S}(152 \mathrm{eV})$ $v s$. sputtering time are plotted in Fig. 2. To obtain the number of atoms of each component in the successive atomic layers by using Eq. (2), the curve of each component in Fig. 2 is divided by the vertical lines into the thickness of monolayer, which corresponds to the sputtering time of $2 \mathrm{~min}$; The sputtering rates of passive films on 304- and 316-stainless steels were not measured but assumed to be approximately equal to those on $\mathrm{Fe}-30 \mathrm{Cr}(0.15 \mathrm{~nm} / \mathrm{min})$. Figures 3 and 4 show the differential composition profiles expressed in terms of the number of component atoms. Thermodynamically, the surface layer may be characterized by the surface excess of component I, $\Gamma_{I}$, which can be easily obtained from the composition profile across the interface between two different homogeneous aqueous and solid phases.

For the estimation of surface excess of alloying and environmental components, a dividing surface is so chosen that there may be no surface excess of iron, which is the major component of the alloy substrate. The dot-dash-line (- - ) drawn vertically in Figs. 3 and 4 designates the dividing surface where $\Gamma_{\mathrm{Fe}}$ is approximately zero. This dividing surface is located between the third and the fourth surface atomic layer. $\Gamma_{I}$ on the dividing surface can be estimated from the summation of $\delta n_{I}$ shown by the arrow-lines $(\downarrow)$ vertically drawn in Figs. 3 and 4. The horizontal lines, $n_{\mathrm{H}_{2} \mathrm{O}}$ and $n_{\mathrm{H}_{2} \mathrm{SO}_{4}}$, in Fig. 4 represent respectively the number densities of water molecules (i.e., oxygen atoms) and sulfur atoms in aqueous phase. The values (atoms $/ \mathrm{cm}^{2}$ ) of $n_{\mathrm{H}_{2} \mathrm{O}}$ and $n_{\mathrm{H}_{2} \mathrm{SO}_{4}}$ obtained from the specific gravity of $0.5 \mathrm{M}$ sulfuric acid solution, is converted into the arbitrary unit (a.u.) with the conversion coefficient calculated by assuming that the number of total metal atoms, $\Sigma n_{\mathrm{Me}}$ in an atomic layer deeper than the 8th layer from the uppermost surface layer, is equal to that in the bulk 316-stainless steel $\left(2.56 \times 10^{15}\right.$ atoms $\left./ \mathrm{cm}^{2}\right)$.

The values of $\Gamma_{\mathrm{Cr}}, \Gamma_{\mathrm{Ni}}, \Gamma_{\mathrm{O}}$ and $\Gamma_{\mathrm{s}}$ thus obtained for $\mathrm{Fe}-30 \mathrm{Cr}$ alloy, 304- and 316-stainless steels are

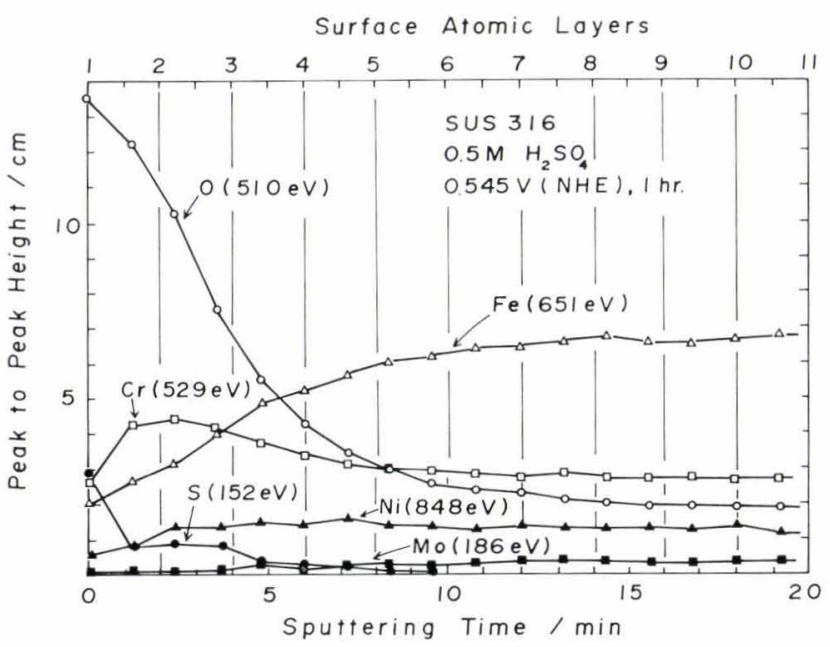

Fig. 2. Peak to peak height of each component vs. sputtering time plotted as a function of anodic potential in Figs. 5 and 6 . The surface excess changes depending on the species of alloys. In special, the surface excess of chromium and oxygen on 316-stainless steel is smaller than that on 304-stainless steel, indicating that molybdenum affects $\Gamma_{\mathrm{Cr}}$ and $\Gamma_{\mathrm{O}}$. The effect of al-

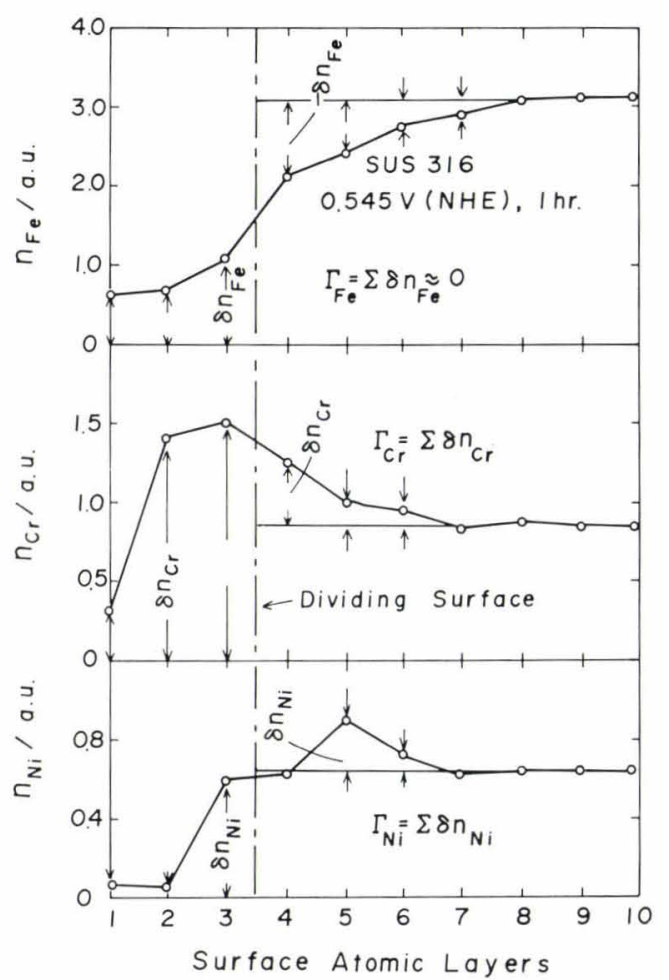

Fig. 3. Differential composition profile in depth expressed in terms of the number of atoms

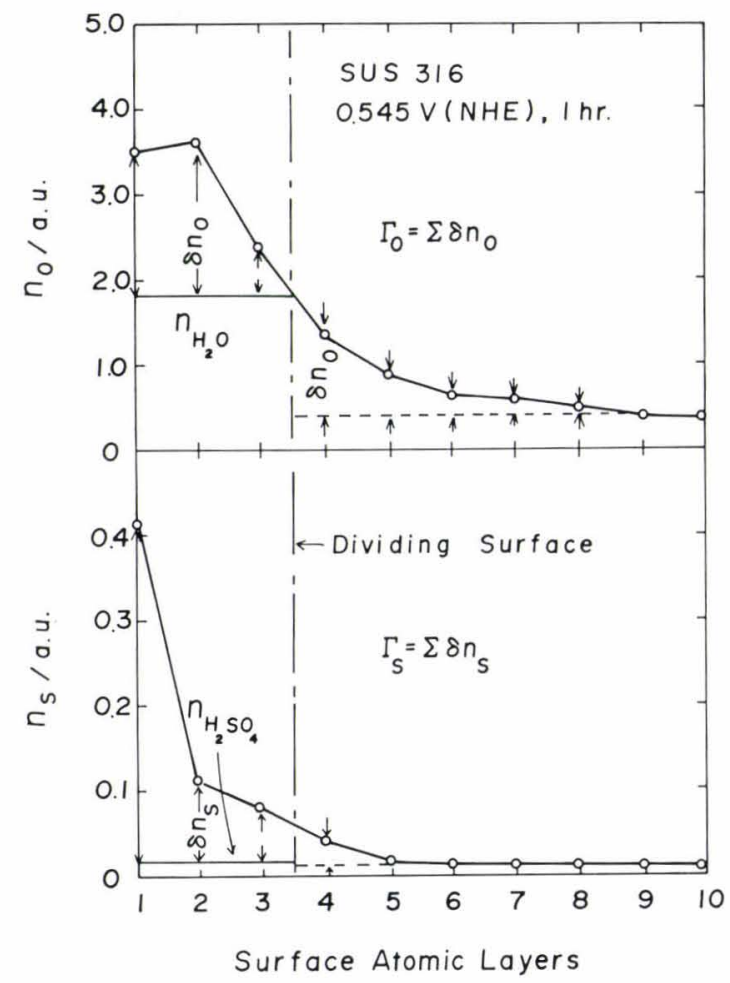

Fig. 4. Differential composition profile in depth expressed in terms of the number of atoms 


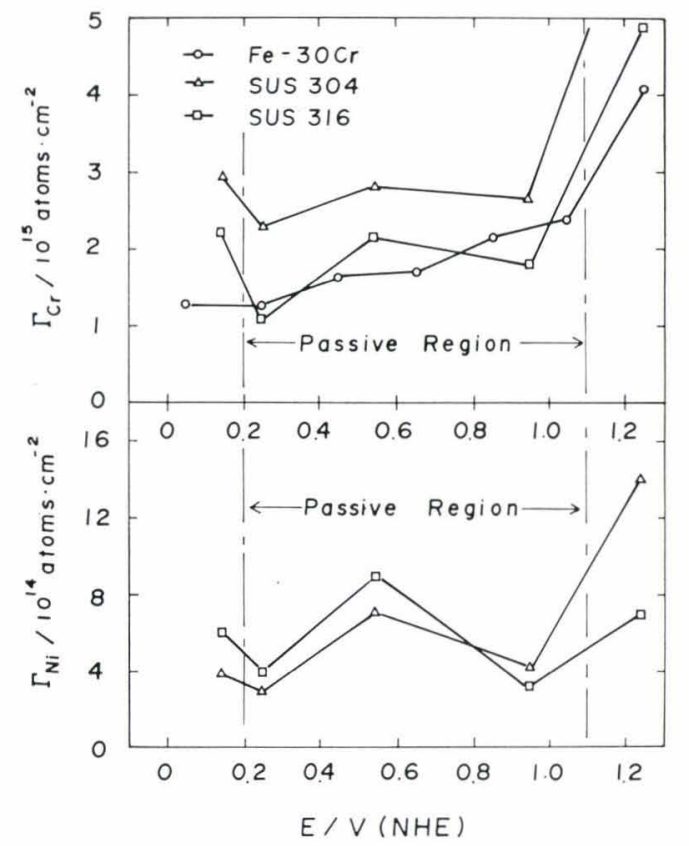

- $\mathrm{O}$ - Fe-30Cr alloy

$-\triangle$ - 304-stainless steel

- $\square$ - 316-stainless steel

Fig. 5. Surface excess, $\Gamma_{\mathrm{Cr}}$ and $\Gamma_{\mathrm{Ni}}$ as a function of anodic potential

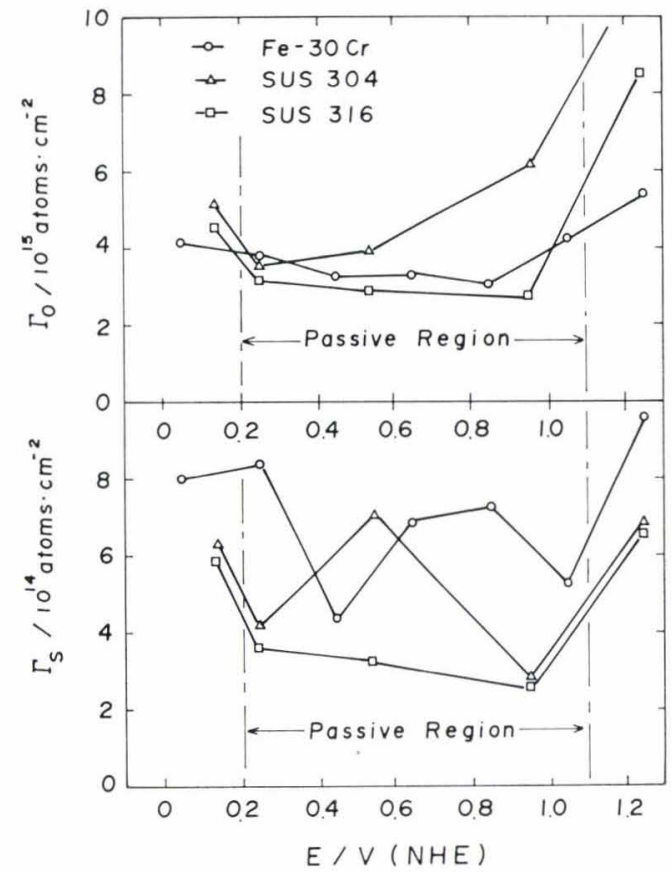

- $-\mathrm{Fe}-30 \mathrm{Cr}$ alloy

$-\triangle-\quad 304$-stainless steel

$-\square-316$-stainless steel

Fig. 6. Surface excess, $\Gamma_{\mathrm{O}}$ and $\Gamma_{\mathrm{S}}$ as a function of anodic potential
Fig. 7.

Polarization curves of 316- and 304-stainless steels measured by means of potential-step method

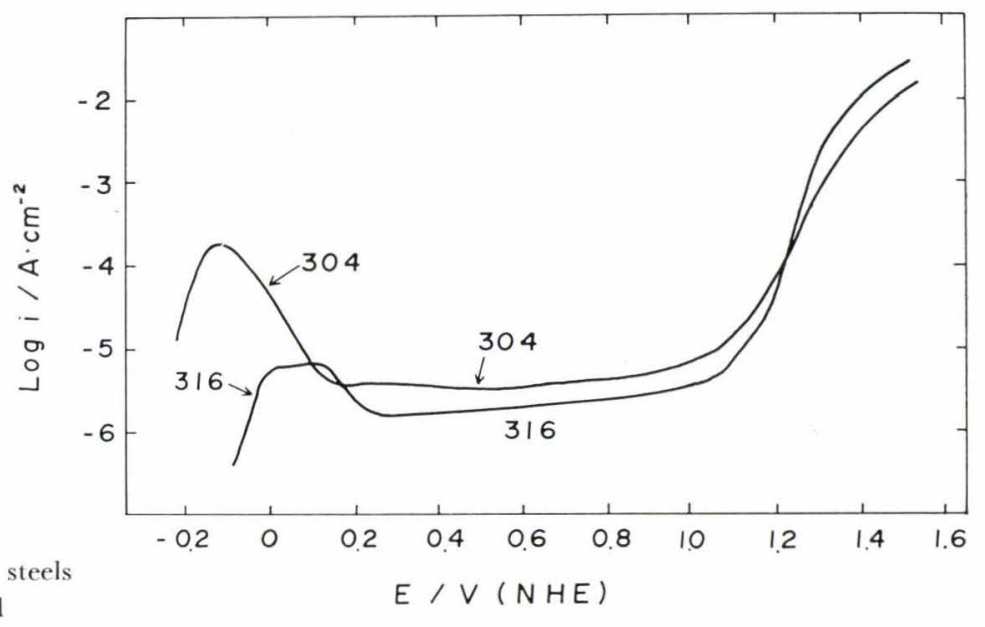

loying molybdenum on $\Gamma_{\mathrm{Cr}}$ and $\Gamma_{\mathrm{O}}$ may be explained by the electrochemical characteristics of molybdenum during passivation. Figure 7 shows the polarization curves of 304- and 316-stainless steels in $0.5 \mathrm{M}$ sulfuric acid solution measured by means of potential step method. Obviously, molybdenum depresses both the active dissolution current $i_{d}$ and the passivity-maintaining current $i_{p}$. It is known ${ }^{9)}$ that iron component preferentially dissolves into sulfuric acid solution from iron-chromium alloys in the active and passive potential region. The depression of $i_{d}$ and $i_{p}$ due to molybdenum, therefore, would give rise to a decrease in $\Gamma_{\mathrm{Cr}}$. Moreover, from the relation between film thickness $\left(\Gamma_{\mathrm{O}}\right)$ and $i_{p}$, it appears that the passive film on 316-stainless steel is more resistive than that on 304-stainless steel against ionic current, provided that the ionic current through the film obeys a field-assisted ion migration mechanism. ${ }^{10)}$ As shown in Fig. 2, no surface enrichment of molybdenum was observed in the passive film on 316-stainless steel. Some authors ${ }^{11)}$ have reported that molybdenum promotes the surface enrichment of chromium, improving the corrosion resistivity. The present results, however, are not consistent with their results. Microscopically, the passive film is always broken and repaired repeatedly as pointed out by noise-analysis ${ }^{12}$ ) of passive film. This suggests that the corrosion resistivity of metals and alloys will have to be discussed from the two sides of micro-breakdown of the film and repassivation of the substrate alloy at breakdown sites. The breakdown of the film depends mainly on the properties of film itself whereas the repassivation depends mainly on the alloy substrate. Evidently, the present results indicate that molybdenum pro- 
motes the repassivation of micro-breakdown sites and enhances the corrosion resistivity.

\section{Summary}

A differential method for obtaining the exact composition profiles in depth was applied to the surface analyses of $\mathrm{Fe}-30 \mathrm{Cr}$ alloy, 304- and 316-stainless steels which were passivated potentiostatically in deaerated $0.5 \mathrm{M}$ sulfuric acid solution. From the exact composition profiles in depth, the surface excess $\Gamma_{I}$ of component, chromium, nickel, oxygen and sulfur on the dividing surface with $\Gamma_{\mathrm{Fe}} \approx 0$ was estimated as a function of anodic potential. The surface excess changes depending on the species of the alloys. In special, the surface excess of chromium and oxygen on 316-stainless steel is smaller than that on 304stainless steel. The effect of molybdenum on $\Gamma_{\mathrm{Cr}}$ and $\Gamma_{\mathrm{o}}$ was discussed with reference to the electrochemical characteristics of molybdenum. Finally, it was concluded from these results that the beneficial effect of molybdenum on the corrosion resistivity of the alloys can not be attributed to the film-composition but to the repassivation of the substrate alloys at breakdown sites.

\section{REFERENCES}

1) J. B. Lumsden and R. W. Staehie: Scr. Metall., 6, (1972), 1205.

2) F. Pons, J. Le Héricy and J. P. Langeron: Surface Sci., 69, (1977), $547 \& 565$.

3) M. da Cunha Belo, B. Rondot, F. Pons, J. Le Héricy and J. P. Langeron: J. Electrochem. Soc., 124 (1977), 1317.

4) M. Seo and N. Sato: Corrosion Eng. (Boshoku Gijitsu), 27, (1978), 172.

5) R.J. Archer: Proc. of Symp. on Ellipsometry in the Measurement of Surface and Thin Films, Nat. Bur. Stand., Washington, Misc. Pub. 256, (1964), 255.

6) H. Kanter: Phys. Rev. B1, (1970), 2357.

7) M. Seo, J. B. Lumsden and R. W. Staehle: Surface Sci, 50, (1975), 541.

8) L. E. Davis, N. C. MacDonald, P. W. Palmberg, G. E. Riach and R. E. Weber: Hand Book of Auger Electron Spectroscopy, ed., Physical Electronics Industries, Inc., Minnesota, (1976).

9) Ya. M. Kolotyrkin: J. Res. Inst. Catalysis, Hokkaido Univ., 22, (1974), No. 2, 73.

10) N. Sato and T. Noda: Electrochem. Acta, 22, (1977), 839.

11) H. Ogawa, H. Omata, I. Itoh and H. Okada: Corrosion, 34, (1978), No. 2, 52.

12) G. Okamoto, T. Sugita, S. Nishiyama and K. Tachibana: Corrosion Eng. (Boshoku Gijutsu), 23, (1974), 439 \& 445; Passivity and Its Breakdown of Iron and Iron-base Alloys, NACE, Houston, (1976), p. 106. 